\title{
CONTINUUM FAMILIES OF NON-DISPLACEABLE LAGRANGIAN TORI IN $\left(\mathbb{C} P^{1}\right)^{2 m}$
}

\author{
RENATO VIANNA
}

\begin{abstract}
We construct a family of Lagrangian tori $\Theta_{s}^{n} \subset\left(\mathbb{C} P^{1}\right)^{n}, s \in(0,1)$, where $\Theta_{1 / 2}^{n}=\Theta^{n}$, is the monotone twist Lagrangian torus described in [6]. We show that for $n=2 m$ and $s \geq 1 / 2$ these tori are non-displaceable. Then by considering $\Theta_{s_{1}}^{k_{1}} \times \cdots \times$ $\Theta_{s_{l}}^{k_{l}} \times\left(S_{\text {eq }}^{2}\right)^{n-\sum_{i} k_{i}} \subset\left(\mathbb{C} P^{1}\right)^{n}$, with $s_{i} \in[1 / 2,1)$ and $k_{i} \in 2 \mathbb{Z}_{>0}, \sum_{i} k_{i} \leq n$ we get several $l$ dimensional families of non-displaceable Lagrangian tori. We also show that there exists partial symplectic quasi-states $\zeta_{\mathbf{e}_{s}}^{\mathfrak{b}_{s}}$ and linearly independent homogeneous Calabi quasimorphims $\mu_{\mathbf{e}_{s}}^{\mathfrak{b}_{s}}$ 15] for which $\Theta_{s}^{2 m}$ are $\zeta_{\mathbf{e}_{s}}^{\mathfrak{b}_{s}}$-superheavy and $\mu_{\mathbf{e}_{s}}^{\mathfrak{b}_{s}}$-superheavy. We also prove a similar result for $\left(\mathbb{C} P^{2} \# 3 \overline{\mathbb{C} P^{2}}, \omega_{\epsilon}\right)$, where $\left\{\omega_{\epsilon} ; 0<\epsilon<1\right\}$ is a family of symplectic forms in $\mathbb{C} P^{2} \# 3 \overline{\mathbb{C} P^{2}}$, for which $\omega_{1 / 2}$ is monotone.
\end{abstract}

\section{INTRODUCTION}

In [16], Fukaya-Oh-Ohta-Ono construct a one-dimensional family of non-displaceable Lagrangian tori in $\left(\mathbb{C} P^{1}\right)^{2}$. They arise as fibres of a (informally called) semi-toric moment map [24, Section 3], where the fibres over the interior of the semi-toric moment polytope are Lagrangian tori, but over a special vertex of the polytope lies a Lagrangian $S^{2}$ (the anti-diagonal) where the semi-toric moment map is not differentiable.

The weighted barycentre of the semi-toric polytope was proven by Oakley-Usher [19] to be the Chekanov torus [6] in $\left(\mathbb{C} P^{1}\right)^{2}$. The other regular fibres are Hamiltonian isotopic to so called Chekanov type tori described in [1, Example 3.3.1]. In fact, the semi-toric Lagrangian fibration described in [16] can be seen as a limit of almost toric fibrations, in which 'most of the fibres' are Chekanov type tori, see [23, Section 6.4] and [20, Remark 3.1].

The definition of Chekanov type tori can be easily extended to higher dimensions, see Definition 4.1. In particular, we can get analogues of the non-displaceable tori [16]. We can show that these tori are non-displaceable in $\left(\mathbb{C} P^{1}\right)^{2 m}$.

\subsection{Results.}

Theorem 1.1. For a positive even integer $n=2 m$, there is a continuum of non-displaceable Lagrangian tori $\Theta_{s}^{2 m} \subset\left(\mathbb{C} P^{1}\right)^{2 m}, s \in[1 / 2,1)$, for which $\Theta_{1 / 2}^{2 m}=\Theta^{2 m}$ is the monotone twist Lagrangian torus described in [6]. More precisely, for any Hamiltonian $\Psi \in \operatorname{Ham}\left(\left(\mathbb{C} P^{1}\right)^{2 m}\right)$, we have that $\left|\Theta_{s}^{2 m} \cap \Psi\left(\Theta_{s}^{2 m}\right)\right| \geq 2^{2 m}$.

The case $n=2$ was proven in [16]. The case $n=1$ is clearly false, since only the monotone circle is non-displaceable.

Question 1.2. For $n \geq 3$ odd and $s \in\left[1 / 2,1\right.$ ), are the tori $\Theta_{s}^{n}$ from Definition 4.2 (non)displaceable?

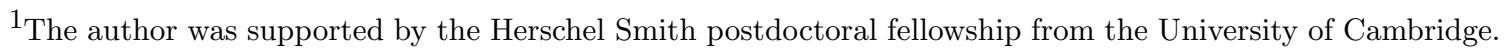


An immediate consequence of the proof of Theorem 1.1 is

Corollary 1.3. For $s_{i} \in[1 / 2,1)$, and positive even integers $k_{i}, i=1, \ldots, l$, and $n \geq \sum_{i} k_{i}$, the Lagrangian tori

are non-displaceable.

$$
\Theta_{s_{1}}^{k_{1}} \times \cdots \times \Theta_{s_{l}}^{k_{l}} \times\left(S_{\text {eq }}^{1}\right)^{n-\sum_{i} k_{i}} \subset\left(\mathbb{C} P^{1}\right)^{n}
$$

Just by looking to the symplectic area spectrum of Maslov index 2 relative homology classes we can conclude:

Proposition 1.4. The tori $\Theta_{s}^{n}$ is not symplectomorphic to $\Theta_{s_{1}}^{k_{1}} \times \cdots \times \Theta_{s_{l}}^{k_{l}} \times\left(S_{\text {eq }}^{1}\right)^{n-\sum_{i} k_{i}}$, if $n>\sum_{i} k_{i}$.

Consider the counts of holomorphic (for the standard complex structure in $\left(\mathbb{C} P^{1}\right)^{n}$ ) Maslov index 2 disks with boundary in $\Theta_{s}^{n}$, respectively $\Theta_{s_{1}}^{k_{1}} \times \cdots \times \Theta_{s_{l}}^{k_{l}}\left(n=\sum_{i} k_{i}\right)$, passing through a fixed point. Among these, look at the count of disks that have minimal area. For $s, s_{i} \in(1 / 2,1)$, this area is $a=1-s$, respectively $1-s_{i}$ for some $i \in\{1, \ldots, l\}$. It follows from Proposition 4.5 that these counts of disks of smaller area are different if $l>1$. Moreover, we show in Proposition 4.10 that higher Maslov index holomorphic disks with boundary on $\Theta_{s}^{n}$ must have symplectic area bigger than $a$. Hence, one expect that in a generic family $J_{t}$ of almost complex structures, where $J_{0}$ is the standard complex structure and $J_{1}$ is another regular almost complex structure, $J_{t}$-holomorphic disks of positive Maslov index and area smaller than a can only appear in a "birth-death" phenomenon. This should imply that the count of Maslov index 2 disks of symplectic area $a$ with boundary in $\Theta_{s}^{n}$ is an invariant under generic choice of almost complex structure, and hence under symplectomorphisms (in particular Hamiltonian isotopies) acting on $\Theta_{s}^{n}$. This would allow us to prove:

Conjecture 1.5. The tori $\Theta_{s}^{n}$ is not symplectomorphic to $\Theta_{s_{1}}^{k_{1}} \times \cdots \times \Theta_{s_{l}}^{k_{l}}, n=\sum_{i} k_{i}-$ unless $l=1$ and $s_{1}=s$.

A rigorous statement proving the invariance of the count of the Maslov index 2 disks of minimal area in the above scenario and hence Conjecture 1.5 is expected to appear in the forthcoming working of the author together with Egor Shelukhin and Dmitry Tonkonog.

Therefore we see that - up to a formal proof of Conjecture 1.5 - the tori obtained here differ from products of copies of the tori obtained in [16] and copies of the equator in $\mathbb{C} P^{1}$.

The idea of the proof of Theorem 1.1 is that we are able to find bulk deformations $\mathfrak{b}_{s}$ for which the bulk deformed Floer Homology of $\Theta_{s}^{2 m}$ (decorated with some weakly bounding cochain $\sigma$ ) is non-zero. The invariance property of the bulk deformed Floer Cohomology under the action of Hamiltonian diffeomorphisms [14, Theorem 2.5], allow us to conclude that the above Lagrangian tori are non-displaceable.

Based on the work of Fukaya-Oh-Ohta-Ono [15], regarding spectral invariants with bulk deformations, quasimorphisms and Lagrangian Floer theory, we are able to strengthen our result and find families of homogeneous Calabi quasimorphisms $\mu_{\mathbf{e}_{s}}^{\mathfrak{b}_{s}}$ and partial symplectic

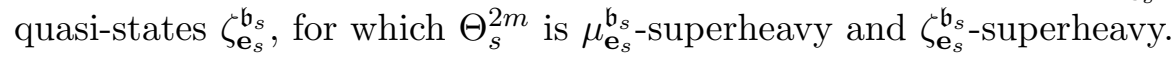

For the definition of homogeneous Calabi quasimorphisms, partial symplectic quasi-states and the notion of superheaviness we refer the reader to [9, 10, 15].

Following closely the notation of [15, Lemma 23.3, Theorem 23.4] we summarise the above discussion as: 
Theorem 1.6. For $s \in[1 / 2,1)$, there exists a bulk-deformation $\mathfrak{b}_{s} \in H^{2}\left(\left(\mathbb{C} P^{1}\right)^{2 m}, \Lambda_{+}\right)$, and a weak bounding cochain $b_{s} \in H^{1}\left(\Theta_{s}^{2 m}, \Lambda_{0}\right)$ for which

$$
H F\left(\Theta_{s}^{2 m},\left(\mathfrak{b}_{s}, b_{s}\right) ; \Lambda_{0, \text { nov }}\right) \cong H^{*}\left(\Theta_{s}^{2 m} ; \Lambda_{0, \text { nov }}\right)
$$

Moreover, there are idempotents $\boldsymbol{e}_{s}$ in the bulk-deformed quantum-cohomology $Q H_{\mathfrak{b}_{s}}^{*}\left(\left(\mathbb{C} P^{1}\right)^{2 m} ; \Lambda_{0, \text { nov }}\right)$,

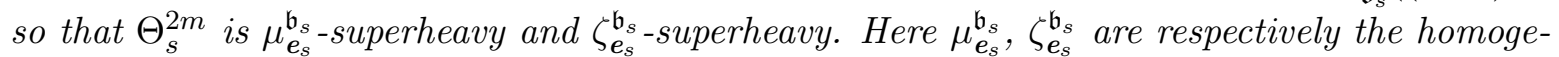
neous Calabi quasimorphism and partial symplectic quasi-states coming from the bulk-deformed spectral invariant associated with $\boldsymbol{e}_{s}[15$, Section 14].

Here $\Lambda, \Lambda_{0}, \Lambda_{n o v}, \Lambda_{0, n o v}$ and $\Lambda_{+}$are the Novikov rings:

$$
\begin{gathered}
\Lambda=\left\{\sum_{i \geq 0} a_{i} T^{\lambda_{i}} \mid a_{i} \in \mathbb{C}, \lambda_{i} \in \mathbb{R}, \lambda_{i} \leq \lambda_{i+1}, \lim _{i \rightarrow \infty} \lambda_{i}=\infty\right\}, \\
\Lambda_{0}=\left\{\sum_{i \geq 0} a_{i} T^{\lambda_{i}} \mid a_{i} \in \mathbb{C}, \lambda_{i} \in \mathbb{R}_{\geq 0}, \lambda_{i} \leq \lambda_{i+1}, \lim _{i \rightarrow \infty} \lambda_{i}=\infty\right\}, \\
\Lambda_{\text {nov }}=\left\{\sum_{i \geq 0} a_{i} q^{n_{i}} T^{\lambda_{i}} \mid n_{i} \in \mathbb{Z} a_{i} \in \mathbb{C}, \lambda_{i} \in \mathbb{R}, \lambda_{i} \leq \lambda_{i+1}, \lim _{i \rightarrow \infty} \lambda_{i}=\infty\right\}, \\
\Lambda_{0, \text { nov }}=\left\{\sum_{i \geq 0} a_{i} q^{n_{i}} T^{\lambda_{i}} \mid n_{i} \in \mathbb{Z} a_{i} \in \mathbb{C}, \lambda_{i} \in \mathbb{R} \geq 0, \lambda_{i} \leq \lambda_{i+1}, \lim _{i \rightarrow \infty} \lambda_{i}=\infty\right\}, \\
\Lambda_{+}=\left\{\sum_{i \geq 0} a_{i} T^{\lambda_{i}} \mid a_{i} \in \mathbb{C}, \lambda_{i} \in \mathbb{R}_{>0}, \lambda_{i} \leq \lambda_{i+1}, \lim _{i \rightarrow \infty} \lambda_{i}=\infty\right\},
\end{gathered}
$$

The formal parameter $T$ is used to keep track of area of pseudo-holomorphic disks, while the formal parameter $q \in \Lambda_{0, \text { nov }}$ is used to keep track of the Maslov index.

The following Corollary follows immediately from [15, Corollary 1.10], see [15, Section 19] for a proof.

Corollary 1.7. The uncountable set $\left\{\mu_{e_{s}}^{\mathfrak{b}_{s}}\right\}$ of homogeneous Calabi quasimorphisms is linearly independent [15, Definition 1.9].

To prove linear independency of the above homogeneous Calabi quasimorphisms we use that the tori are disjoint, for different values of $s$. One could ask:

Question 1.8. Are the tori $\Theta_{s}^{n}$ Hamiltonian displaceable from $\Theta_{s_{1}}^{k_{1}} \times \cdots \times \Theta_{s_{l}}^{k_{l}} \times\left(S_{\text {eq }}^{1}\right)^{n-\sum_{i} k_{i}}$, for $s, s_{i} \in(1 / 2,1)$ ?

We note that by construction, these tori intersect for $s, s_{i} \geq 1 / 2$. See [20], for nondisplaceability in the case $n=2$, between $\Theta_{s}^{n}$ (i.e. tori from [16]) $s \geq 3 / 2$ and the Clifford torus $S_{\text {eq }}^{1} \times S_{\text {eq }}^{1}$.

Question 1.9. Are the quasimorphisms arising from (particular choice of bulk-deformation and weak-bounding cochain for) the tori in Corollary 1.3 linearly independent for different partitions $\left(k_{1}, \ldots, k_{l}, n-\sum_{i} k_{i}\right)$ of $n$ ? 
We finish our results by pointing out that the family given in [16] remain non-displaceable after we perform two blowups (of the same size) on the rank zero corners of the singular fibration described in [16], see Figure 11. This follows from applying the same ideas as Fukaya-Oh-Ohta-Ono did for the $\mathbb{C} P^{1} \times \mathbb{C} P^{1}$ case.

Theorem 1.10. There exists a continuous family of non-displaceable Lagrangian tori $L_{s}^{\epsilon}$ in $\left(\mathbb{C} P^{2} \# 3 \overline{\mathbb{C} P^{2}}, \omega_{\epsilon}\right)=\left(\mathbb{C} P^{1} \times \mathbb{C} P^{1} \# 2 \overline{\mathbb{C} P^{2}}, \omega_{\epsilon}\right)$, where $s \in[1 / 2,1)$ and $\left\{\omega_{\epsilon} \mid 0<\epsilon<1\right\}$ is a family of symplectic forms for which $\left(\mathbb{C} P^{2} \# 3 \overline{\mathbb{C} P^{2}}, \omega_{1 / 2}\right)$ is monotone, containing a monotone Lagrangian $L_{1 / 2}^{1 / 2}$.

Remark 1.11. It is shown in [14, Section 5] and [15, Section 22] a family of non-displaceable Lagrangian tori in $\mathbb{C} P^{2} \# k \overline{\mathbb{C} P^{2}}, k \geq 2$, endowed with some non-monotone symplectic form.

Theorem 1.10 follows, in the same spirit as [15, Theorem 1.11] and Theorem 1.6, from:

Theorem 1.12. Let $\left(\mathbb{C} P^{2} \# 3 \overline{\mathbb{C} P^{2}}, \omega_{\epsilon}\right)$ and $L_{s}^{\epsilon}$ be as in Theorem 1.10. For $s \in[1 / 2,1)$, there exists a bulk-deformation $\mathfrak{b}_{s}^{\epsilon} \in H^{2}\left(\mathbb{C} P^{2} \# 3 \overline{\mathbb{C} P^{2}}, \Lambda_{+}\right)$, and a weak bounding cochain $b_{s}^{\epsilon} \in H^{1}\left(L_{s}^{\epsilon}, \Lambda_{0}\right)$ for which

$$
H F\left(\mathbb{C} P^{2} \# 3 \overline{\mathbb{C} P^{2}},\left(\mathfrak{b}_{s}^{\epsilon}, b_{s}^{\epsilon}\right) ; \Lambda_{0, n o v}\right) \cong H^{*}\left(L_{s}^{\epsilon} ; \Lambda_{0, \text { nov }}\right)
$$

There are idempotents $\boldsymbol{e}_{s}^{\epsilon}$ in the bulk-deformed quantum-cohomology $Q H\left(\mathbb{C} P^{2} \# 3 \overline{\mathbb{C} P^{2}} ; \Lambda\right)$, so that $L_{s}^{\epsilon}$ is $\mu_{\boldsymbol{e}_{s}^{\epsilon}}^{\mathfrak{b}_{s}^{\epsilon}}$-superheavy and $\zeta_{\boldsymbol{e}_{s}^{\epsilon}}^{\mathfrak{b}_{s}^{\epsilon}}$-superheavy, where $\mu_{\boldsymbol{e}_{s}^{\epsilon}}^{\mathfrak{b}_{s}^{\epsilon}}, \zeta_{\boldsymbol{e}_{s}^{\epsilon}}^{\mathfrak{b}_{s}^{\epsilon}}$ are the homogeneous Calabi quasimorphism and partial symplectic quasi-states coming from the bulk-deformed spectral invariant associated with $\boldsymbol{e}_{s}^{\epsilon}$ [15, Section 14]. Moreover, the uncountable set $\left\{\mu_{\boldsymbol{e}_{s}^{\epsilon}}^{\mathfrak{b}_{s}^{\epsilon}}\right\}$ of homogeneous Calabi quasimorphisms is linearly independent.

The rest of the paper is organised as follows:

In Section 2, we make a quick introduction of bulk deformed potential and Floer cohomology for a Lagrangian $L$ satisfying Assumption 2.1. We refer the reader to [14, 15, 16] for a complete account. We then prove Lemma 2.5 and Corollary 2.8, to show that, for a Lagrangian torus $T$, critical points of the potential gives rise to (bulk deformed) Floer cohomology isomorphic to the usual cohomology of $T$. We believe that 2.5 is known by experts on the field, but we are not aware of it being written.

In Section 3, we define the notion of a pair $(X, L)$ consisting of a Kähler manifold $X$ and a Lagrangian submanifold $L$ being $K$-pseudohomogeneous, for some Lie group $K$ acting holomorphically and Hamiltonianly on $X$, leaving $L$ invariant. We showed that if $(X, L)$ is $K$-pseudohomogeneous, any Maslov index 2 holomorphic disk with boundary on $L$ such that its boundary is transverse to $K$-orbits, is regular. We use that to show regularity for the Maslov index 2 disks with boundary in $\Theta_{s}^{n}$.

In Section 4, we define the Lagrangian tori $\Theta_{s}^{n}$, establish its potential function, essentially computed in [1, 2], and prove it satisfies Assumption 2.1, for some regular almost complex structure $J$ with the same potential function of the standard complex structure. We also prove Proposition 1.4 and show that holomorphic disks of Maslov index bigger than 2 have area bigger than $a=1-s$, which we use to argue why Conjecture 1.5 should hold.

In Section 5, we compute the critical points of the potential bulk deformed by some cocycle in $C^{2}\left(\left(\mathbb{C} P^{1}\right)^{n}, \Lambda_{+}\right)$. We show that for $n=2 m$, there are bulk deformation $\mathfrak{b}_{s}$ and a weak bounding cochain $b_{s}$ which is a critical point of the potential $\mathfrak{P O}_{\mathfrak{b}_{s}}^{\Theta_{s}^{2 m}}$. It then follows from Corollary 2.8 that the bulk deformed Floer cohomology $H F\left(\Theta_{s}^{2 m},\left(b_{s}, \mathfrak{b}_{s}\right) ; \Lambda\right)$ is isomorphic to 
the cohomology of the torus. Non-displaceability then follows from [13, Theorem G] which is also stated as [14, Theorem 2.5].

In Section 6, we finish the proof of Theorem 1.6.

Finally in Section 7, we describe $\left(\mathbb{C} P^{2} \# 3 \overline{\mathbb{C} P^{2}}, \omega_{\epsilon}\right)=\left(\mathbb{C} P^{1} \times \mathbb{C} P^{1} \# 2 \overline{\mathbb{C} P^{2}}, \omega_{\epsilon}\right)$ as two blowups of capacity $\epsilon$ on two corners of the moment polytope of $\mathbb{C} P^{1} \times \mathbb{C} P^{1}$. The Lagrangian tori $L_{s}^{\epsilon}$ on the blowup comes from $\Theta_{s}^{2} \in \mathbb{C} P^{1} \times \mathbb{C} P^{1}$. We compute the potential for $L_{s}^{\epsilon}$ and show the existence of critical points for some bulk deformation. This allow us to prove Theorems 1.10 and 1.12 . These tori are equivalent to the fibres of the singular fibration given by blowing up the corners of the "semi-toric polytope" described in [16], see Figure 1.

Acknowledgements. We are very grateful to Georgios Dimitroglou Rizell, Ivan Smith, Dmitry Tonkonog and Kaoru Ono for useful discussions.

\section{Floer homology And the potential FunCtion}

Let $X$ be a symplectic manifold and $J$ a regular and compatible almost complex structure. Let $L$ be a Lagrangian submanifold of $X$ (with a chosen spin structure). We consider a unital canonical $A_{\infty}$ algebra structure $\left\{\mathrm{m}_{k}\right\}$ on the classical cohomology $H\left(L ; \Lambda_{0, \text { nov }}\right)$ [16, Section 6], [13. Corollary 5.4.6, Theorem A]. The potential function is defined from the space of weak bounding cochains $\hat{\mathcal{M}}(L)$ of $L$ to $\Lambda_{0}$. We refer the reader to [14, 15, 16, 13] for the definition.

Suppose we are given an compatible almost complex structure $J_{0}$ for which $\left(X, L, J_{0}\right)$ satisfy:

Assumption 2.1. Let $\beta \in \pi_{2}(X, L)$. Assume that:

$\left(A_{1}\right)$ If $\beta$ is represented by a non-constant $J_{0}$-holomorphic disk, then $\mu_{L}(\beta) \geq 2$,

$\left(A_{2}\right)$ Maslov index $2 J_{0}$-holomorphic disks are regular,

where $\mu_{L}$ is the Maslov index.

Throughout the paper we say an almost complex structure $J$ is regular if it satisfies assumption $\left(A_{2}\right)$.

An almost complex structure satisfying Assumption 2.1, automatically satisfies [16, Condition 6.1], hence by [16, Theorem A.1, Theorem A.2] there is an embedding of $H^{1}\left(L, \Lambda_{0}\right)$ into $\hat{\mathcal{M}}(L)$ and restricted to $H^{1}\left(L, \Lambda_{0}\right)$ the potential function $\mathfrak{P O}^{L}$ is so that

$$
\mathrm{m}_{0}^{b}(1)=\mathfrak{P O}^{L}(b) q[L],
$$

where

$$
\mathrm{m}_{0}^{b}(1)=\sum_{k=0}^{\infty} \mathrm{m}_{k}(b, \ldots, b)=\sum_{\substack{\beta \in \pi_{2}(X, L), \mu_{L}(\beta)=2}} q^{\mu_{L}(\beta) / 2} T^{\int_{\beta} \omega} \exp (b \cap \partial \beta) \operatorname{ev}_{0 *}\left(\left[\mathscr{M}_{1}(\beta)\right]\right) .
$$

Here $\left[\mathscr{M}_{1}(\beta)\right]$ is the (virtual) fundamental class of the moduli space of $J$-holomorphic disks in the class $\beta$ with 1 marked point and $\mathrm{ev}_{0}: \mathscr{M}_{1}(\beta) \rightarrow L$ is the evaluation map.

Using a notation closer to [1, 2] we define for $\beta \in \pi_{2}(X, L)$ :

$$
z_{\beta}(L, b)=T^{\int_{\beta} \omega} \exp (b \cap \partial \beta) .
$$

Letting $\eta_{\beta}$ be the degree of $\operatorname{ev}_{0}: \mathscr{M}_{1}(\beta) \rightarrow L$, we can write:

$$
\mathfrak{P O}^{L}(b)=\sum_{\substack{\beta \in \pi_{2}(X, L) \\ \mu_{L}(\beta)=2}} \eta_{\beta} z_{\beta}(L, b)
$$


We want to consider the Floer cohomology of $L$ bulk-deformed by a class $\mathfrak{b}=T^{\rho}[\mathfrak{s}] \in$ $H^{2}\left(X, \Lambda_{+}\right)$[14]. The potential function will depend on the cocycle $\mathfrak{b} \in C^{2}(X, L ; \mathbb{Z})$, even though the Floer cohomology doesn't. Since we use a cocycle in degree 2 (Poincaré dual to a cycle of codimension 2) the degree of the bulked deformed $A_{\infty}$ maps $\mathrm{m}_{k}^{\mathfrak{b}}$ [14, (2.6)] is unaffected by the bulk and the bulk deformed potential is given by:

$$
\mathfrak{P O}_{\mathfrak{b}}^{L}(b)=\sum_{\substack{\beta \in \pi_{2}(X, L), \mu_{L}(\beta)=2}} \eta_{\beta} \exp \left[(\mathfrak{s} \cap \beta) T^{\rho}\right] z_{\beta}(L, b),
$$

where $b \in H^{1}\left(L, \Lambda_{0}\right)$, is a weak bounding cochain for the curved $A_{\infty}$ algebra $\left(H\left(L, \Lambda_{0, n o v}\right),\left\{\mathrm{m}_{k}^{\mathfrak{b}}\right\}\right)$, with

$$
\mathrm{m}_{0}^{b, \mathfrak{b}}(1)=\sum_{k=0}^{\infty} \mathrm{m}_{k}^{\mathfrak{b}}(b, \ldots, b)=\mathfrak{P O}_{\mathfrak{b}}^{L}(b) q[L] .
$$

The fact that $b \in H^{1}\left(L, \Lambda_{0}\right)$ is a weak bounding cochain for $\left(H\left(L, \Lambda_{0}\right),\left\{\mathrm{m}_{k}^{\mathfrak{b}}\right\}\right)$ implies that we can define a (not curved) $A_{\infty}$ algebra $\left(H\left(L, \Lambda_{0, n o v}\right),\left\{\mathrm{m}_{k}^{b, \mathfrak{b}}\right\}\right)$, where

$$
\mathrm{m}_{k}^{b, \mathfrak{b}}\left(x_{1}, \ldots, x_{k}\right)=\sum_{j=0}^{\infty} \mathrm{m}_{j}^{\mathfrak{b}}\left(b, \ldots, b, x_{1}, b, \ldots, b, x_{2}, b, \ldots, b, x_{k}, b, \ldots, b\right) .
$$

In particular,

$$
\begin{gathered}
\left(\mathrm{m}_{1}^{b, \mathfrak{b}}\right)^{2}=0 \\
\mathrm{~m}_{1}^{b, \mathfrak{b}}\left(\mathrm{m}_{2}^{b, \mathfrak{b}}(x, y)\right)= \pm \mathrm{m}_{2}^{b, \mathfrak{b}}\left(\mathrm{m}_{1}^{b, \mathfrak{b}}(x), y\right) \pm \mathrm{m}_{2}^{b, \mathfrak{b}}\left(x, \mathrm{~m}_{1}^{b, \mathfrak{b}}(y)\right) .
\end{gathered}
$$

Definition 2.2. We define the bulk deformed Floer cohomology:

$$
H F\left(L,(b, \mathfrak{b}) ; \Lambda_{0, \text { nov }}\right)=\frac{\operatorname{ker}\left(\mathrm{m}_{1}^{b, \mathfrak{b}}\right)}{\operatorname{im}\left(\mathrm{m}_{1}^{b, \mathfrak{b}}\right)}
$$

Remark 2.3. Strengthening Assumption 2.1 to assume regularity of holomorphic disks with Maslov index smaller than $n-1$, one should be able to define the Floer cohomology using the Pearl version [3], and analogously define its bulk-deformed version, which should be isomorphic to the one in Definition 2.2. In that framework, the proof of Leibniz rule 2.9$)$ follows the same ideas as [5, Theorem 4].

By the work of Fukaya-Oh-Ohta-Ono, we have:

Theorem 2.4 ( Theorem G [13], Theorem 2.5 [14]). If $\psi: X \rightarrow X$ is a Hamiltonian diffeomorphism, then the order of $\psi(L) \cap L$ is not smaller than the rank of $H F\left(L,(b, \mathfrak{b}) ; \Lambda_{0, \text { nov }}\right) \otimes_{\Lambda_{0, \text { nov }}}$ $\Lambda_{\text {nov }}$.

We would like to point out that the product $\mathrm{m}_{2}^{b, \mathfrak{b}}$ can be thought as deformation of the cup product in the sense that for $x, y \in H\left(L, \Lambda_{0}\right)$ of pure degrees $|x|$ and $|y|$,

$$
\mathrm{m}_{2}^{b, \mathfrak{b}}(x, y)=x \cup y+\text { other terms }
$$

where $x \cup y$ comes from counting constant disks and the other terms is a sum of elements of degree smaller than $|x|+|y|$ in $H\left(L, \Lambda_{0, n o v}\right)$, since it comes from evaluating moduli spaces $\mathscr{M}_{k, l+1}(\beta)$ to a cycle of dimension $|x|+|y|-\mu_{L}(\beta)$ and $(X, L, J)$ satisfies Assumption $\left(A_{1}\right)$. 
The following Lemma is well established for the monotone case in [5], and in the general case in [16].

Lemma 2.5 (Theorem 2.3 of [16]). Take $(X, L)$ satisfying Assumption 2.1. Also assume that $H\left(L, \Lambda_{0}\right)$ is generated by $H^{1}\left(L, \Lambda_{0}\right)$ as an algebra with respect to the classical cup product. If $\left.\mathrm{m}_{1}^{b, \mathfrak{b}}\right|_{H^{1}\left(L, \Lambda_{0, \text { nov }}\right)}=0$ then $\mathrm{m}_{1}^{b, \mathfrak{b}} \equiv 0$.

Proof. First we point out that $\left.\mathrm{m}_{1}^{b, \mathfrak{b}}\right|_{H^{0}\left(L, \Lambda_{0, \text { nov }}\right)}=0$. Since $H\left(L, \Lambda_{0}\right)$ is generated by $H^{1}\left(L, \Lambda_{0}\right)$ with respect to the cup product, we only need to show by induction on the degree that for $x$ and $y$ of pure degree $|x| \geq 1,|y| \geq 1, \mathrm{~m}_{1}^{b, \mathfrak{b}}(x \cup y)=0$, if $\mathrm{m}_{1}^{b, \mathfrak{b}}(z)=0$ for all $z$, such that $|z|<|x|+|y|$. Using (2.11),

$$
\mathrm{m}_{1}^{b, \mathfrak{b}}(x \cup y)=\mathrm{m}_{1}^{b, \mathfrak{b}}\left(\mathrm{m}_{2}^{b, \mathfrak{b}}(x, y)\right)-\mathrm{m}_{1}^{b, \mathfrak{b}} \text { (other terms) }=0
$$

by induction hypothesis and using the Leibniz rule 2.9 .

Remark 2.6. Lemma 2.5 strengthen the result of [13, Theorem 6.4.35] and [5], showing that the minimal Maslov number $M_{L}$ of any Lagrangian torus $L$ (or any orientable Lagrangian such that the cohomology ring is generated by $\left.H^{1}\right)$ in $\mathbb{C}^{n}$ is 2 , provided $T$ satisfies Assumption 2.1 for some $J$. That is because the Lagrangian is orientable and $H F(T,(b, \mathfrak{b}) ; \Lambda) \equiv 0$ (from Theorem 2.4. since $T$ is displaceable), so there must be a Maslov index 2 disk. The inequality $2 \leq M_{L} \leq n+1$ was proven in [13, Theorem 6.1.17], for any spin Lagrangian $L \subset \mathbb{C}^{n}$ satisfying Assumption 2.1, via the use of spectral sequence.

Definition 2.7. Take $(X, L)$ satisfying the assumptions of Lemma 2.5. Assume that $\pi_{1}(L) \cong$ $H_{1}(L, \mathbb{Z})$ and $\pi_{2}(X, L) \cong \pi_{2}(X) \oplus H_{1}(L, \mathbb{Z})$. So, we are able to write the Potential function (2.5) in terms of $z_{i}=z_{\beta_{i}}$, for some $\beta_{1}, \ldots, \beta_{n} \in \pi_{2}(X, L)$, where $\partial \beta_{1}, \ldots, \partial \beta_{n}$ is a basis of $H_{1}(L, \mathbb{Z})$. We say that $b$ is a critical point of $\mathfrak{P O}_{\mathfrak{b}}^{L}(b)$ if:

$$
z_{i} \frac{\partial \mathfrak{P O} \mathfrak{O}_{\mathfrak{b}}^{L}(b)}{\partial z_{i}}=0
$$

Corollary 2.8 (Theorem 2.3 of [16]). Take $(X, L)$ satisfying the assumptions of Lemma 2.5 and Definition 2.7. If $b$ is a critical point of $\mathfrak{P O}_{\mathfrak{b}}^{L}(b)$ (2.5) for $\mathfrak{b}=T^{\rho}[\mathfrak{s}] \in H^{2}\left(X, \Lambda_{+}\right)$, then $H F(L,(b, \mathfrak{b}) ; \Lambda) \cong H(L ; \Lambda)$.

Proof. Take a basis $x_{1}, \ldots, x_{n}$ a basis of $H_{1}(L, \mathbb{Z})$. Let $\beta_{1}, \ldots, \beta_{n} \in \pi_{2}(X, L) \cong \pi_{2}(X) \oplus$ $H_{1}(L, \mathbb{Z})$, be so that $\partial \beta_{i}=x_{i} \in H_{1}(L, \mathbb{Z})$ and write the Potential $\mathfrak{P O}_{\mathfrak{b}}^{L}(b)(2.5)$ in terms of $z_{i}=z_{\beta_{i}}$.

Since $\mathfrak{s}$ is of degree 2 , we have that $\mathrm{m}_{1}^{b, \mathfrak{b}}(\sigma)$ for $\sigma \in H^{1}(L, \Lambda)$, only counts contributions of Maslov index 2 disks. A Maslov index $2 J$-holomorphic disk in the class $\beta=\gamma+k_{1} \beta_{1}+\cdots+k_{n} \beta_{n}$, $\gamma \in \pi_{2}(X)$ contributes to $\mathrm{m}_{1}^{b, \mathfrak{b}}(\sigma)$ as

$$
\sum_{i} k_{i}\left(\sigma \cap x_{i}\right) \eta_{\beta} \exp \left[(\mathfrak{s} \cap \beta) T^{\rho}\right] T^{\int_{\gamma} \omega} z_{1}^{k_{1}} \cdots z_{n}^{k_{n}}
$$

Summing all contributions of Maslov index $2 J$-holomorphic disks we have:

$$
\mathrm{m}_{1}^{b, \mathfrak{b}}(\sigma)=\sigma \cap \sum_{i} x_{i}\left(z_{i} \frac{\partial \mathfrak{P \mathcal { O } _ { \mathfrak { b } } ^ { L } ( b )}}{\partial z_{i}}\right)
$$


Therefore, if $b$ is a critical point of $\mathfrak{P O}_{\mathfrak{b}}^{L}(b)$, we have that $\left.\mathrm{m}_{1}^{b, \mathfrak{b}}\right|_{H^{1}(L, \Lambda)}=0$ and by Lemma $2.5 . \mathrm{m}_{1}^{b, \mathfrak{b}} \equiv 0$, so $H F\left(L,(b, \mathfrak{b}) ; \Lambda_{0, \text { nov }}\right) \cong H\left(L ; \Lambda_{0, \text { nov }}\right)$.

\section{Regularity Lemma}

We now move to the Kähler setting and we discuss a Lemma that we will use to prove regularity for Maslov index 2 disks with boundary on $\Theta_{s}^{n}$ with respect to the standard complex structure in $\left(\mathbb{C} P^{1}\right)^{n}$. The following definition is inspired in [11, Definition 1.1.1].

Definition 3.1. Let $L$ be a $n$ dimensional Lagrangian in a Kähler manifold $X$. Assume that $K$ is a Lie group of dimension $n-1$ acting Hamiltonianly and holomorphically on $X$ preserving $L$. Assume that the action restricted to $L$ is free. Then we say that $(X, L)$ is K-pseudohomogeneous.

We get then the following Lemma:

Lemma 3.2. Let $(X, L)$ be $K$-pseudohomogeneous, for some Lie group $K$. If u is a Maslov index 2 holomorphic disk such that $\partial u$ is transverse to the $K$-orbits, then $u$ is regular.

The proof of the above Lemma relies on the Lemmas below, very similar to [21, Lemmas 5.19, 5.20].

Lemma 3.3. Let $u: \mathbb{D} \rightarrow X$ be a Maslov index 2 disk in a Kähler manifold $X$ of complex dimension $n$ with boundary on a Lagrangian L. Assume that $u_{\mid \partial \mathbb{D}}$ is an immersion. Call $W=d u\left(r^{\partial} / \partial \theta\right)$ a holomorphic vector field along $u$ vanishing at 0 and tangent to the boundary. Assume also that there exists $V_{1}, \ldots, V_{n-1}$ holomorphic vector fields in $u^{*} T X$ such that $W \wedge$ $V_{1} \wedge \cdots \wedge V_{n-1} \neq 0$ along the boundary of $u$. Then $u$ is an immersion and no linear combination of the $V_{i}$ 's is tangent to $u(\mathbb{D})$.

Proof. Up to reparametrization, we may assume $d u(0) \neq 0$. The result follows from the fact that the zeros of $\operatorname{det}^{2}\left(W \wedge V_{1} \wedge \cdots \wedge V_{n-1}\right)$ computes the Maslov index, which is assumed to be 2 . So $W \wedge V_{1} \wedge \cdots \wedge V_{n-1}$ can only vanish once (with order 1 ). Since $W$ already vanishes at 0 , we cannot have either $d u(x)=0$ or a linear combination of the $V_{i}$ 's being a complex multiple of $W$.

Lemma 3.4. Let $u_{\theta_{1}, \ldots, \theta_{n-1}}$ be an $n-1$ dimensional family of Maslov index 2 holomorphic disks in a Kähler manifold $X$ of complex dimension $n, \theta_{i} \in(-\epsilon, \epsilon)$. If $u:=u_{0, \ldots, 0}$ and $V_{i}:=\frac{\partial u}{\partial \theta_{i}}$ satisfy the hypothesis of Lemma 3.3, then $u$ is regular.

Proof. It follows similar arguments as in [21, Lemma 5.19]. Using Lemma 3.3, we are able to split $u^{*} T X=T \mathbb{D} \oplus \mathfrak{L}_{1} \oplus \cdots \oplus \mathfrak{L}_{n}$, as holomorphic vector bundles where $\mathfrak{L}_{i}$ is the trivial line bundle generated by $V_{i}$. Also, $u_{\mid \partial \mathbb{D}}^{*} T L=T \partial \mathbb{D} \oplus \operatorname{Re}\left(\mathfrak{L}_{1}\right) \oplus \cdots \oplus \operatorname{Re}\left(\mathfrak{L}_{n}\right)$. As in 21, proof of Lemma 5.19], we see that the kernel of the linearised $\bar{\partial}$ operator is isomorphic to

$$
T_{\mathrm{Id}} \operatorname{Aut}(\mathbb{D}) \bigoplus_{i=1}^{n-1} \operatorname{hol}((\mathbb{D}, \partial \mathbb{D}),(\mathbb{C}, \mathbb{R}))
$$

Hence the kernel has dimension $n+2=n+\mu_{\Theta_{s}^{n}}(u)=$ index. 
Proof of Lemma 3.2. Since the $K$ action is holomorphic and $\partial u$ is transverse to the $K$-orbits, we can build $u_{\theta_{1}, \ldots, \theta_{n}}$ from a neighbourhood of $\operatorname{Id} \in K$, satisfying all the hypothesis of Lemma 3.4 .

\section{The Lagrangian tori $\Theta_{s}^{n}$}

In this section we give an explicit description of the tori $\Theta_{s}^{n}$ and of its potential function, which encodes the number of Maslov index 2 disks that $\Theta_{s}^{n}$ bounds. For a definition of the potential, we refer the reader to [12, Section 4],[13. See also the definition of superpotential in [2, Section 2.2].

The tori $\Theta_{s}^{n}$ appears as fibres of a singular Lagrangian fibration analogous to the one described in [2, Example 3.3.1].

4.1. Definition of $\Theta_{s}^{n}$. Consider $\left(\mathbb{C} P^{1}\right)^{n}$ with the standard symplectic form, for which the symplectic area of each $\mathbb{C} P^{1}$ factor is 1 . For $1 \leq i \leq n$, let $\left[x_{i}: y_{i}\right]$ denote the $i$-th coordinate of $\left(\mathbb{C} P^{1}\right)^{n}$. Consider the function $f=\prod_{i} \frac{x_{i}}{y_{i}}$, defined from the complement of $V=\bigcup_{i, j}\left\{x_{i}=0\right\} \cap\left\{y_{j}=0\right\}$ to $\mathbb{C} P^{1}$, whose fibres are preserved by the $T^{n-1}$ action given by

$$
\begin{aligned}
& \left(\theta_{1}, \ldots, \theta_{n-1}\right) \cdot\left(\left[x_{1}: y_{1}\right], \ldots,\left[x_{n-1}: y_{n-1}\right],\left[x_{n}: y_{n}\right]\right) \\
= & \left(\left[e^{\theta_{1}} x_{1}: y_{1}\right], \ldots,\left[e^{i \theta_{n-1}} x_{n-1}, y_{n-1}\right],\left[e^{-i \sum_{j} \theta_{j}} x_{n}: y_{n}\right]\right),
\end{aligned}
$$

and $\mathrm{m}:\left(\mathbb{C} P^{1}\right)^{n} \rightarrow \mathbb{R}^{n-1}$ its moment map.

Definition 4.1. Let $\gamma$ be an embedded circle on $\mathbb{C}^{\star}$, not enclosing $0 \in \mathbb{C}$, and $\lambda \in \mathbb{R}^{n-1}$. Define the $\Theta^{n}$-type Lagrangian torus:

$$
\Theta_{\gamma, \lambda}^{n}=\left\{x \in\left(\mathbb{C} P^{1}\right)^{n} \backslash V ; f(x) \in \gamma, \mathrm{m}(x)=\lambda\right\}
$$

Noting that $\mathrm{m}^{-1}(0)=\left\{\left|x_{i} / y_{i}\right|=\left|x_{n} / y_{n}\right|, \forall i=1, \ldots, n-1\right\}$, one can see, by using the maximum principle, that $\Theta_{\gamma, 0}^{n}$ bounds only one $(n-1)$-family of holomorphic disks that project injectively to the interior of $\gamma$. Call $\beta_{\gamma} \in \pi_{2}\left(\left(\mathbb{C} P^{1}\right)^{n}, \Theta_{\gamma, 0}^{n}\right)$ the class represented by each of the above disk. We note that there are $n$ disjoint holomorphic disks in the class $\beta_{\gamma}$ inside the line $\Delta=\left\{\left[x_{i}: y_{i}\right]=\left[x_{n}: y_{n}\right], \forall i=1, \ldots, n-1\right\}$. Since $\int_{\Delta} \omega=n$, we see that $\int_{\beta_{\gamma}} \omega \in(0,1)$.

Foliate $\mathbb{C} \backslash \mathbb{R}_{\leq 0}$ by curves $\gamma_{s}, s \in[0,1)$ so that $\gamma_{0}$ is a point, say $1 \in \mathbb{C}$, and for $s \in(0,1)$, $\gamma_{s}$ is an embedded circle so that $\int_{\beta_{\gamma_{s}}} \omega=s$.

Definition 4.2. Define the Lagrangian torus $\Theta_{s}^{n}$ to be $\Theta_{\gamma_{s}, 0}^{n}$.

The hamiltonian isotopy class of $\Theta_{s}^{n}$, does not depend in the curve $\gamma_{s}$ inside $\mathbb{C} \backslash \mathbb{R}_{\leq 0}$, but only on $s=\int_{\beta_{\gamma_{s}}} \omega$.

Consider the divisor $D=f^{-1}(1) \bigcup_{i}\left\{y_{i}=0\right\}$ and the holomorphic $n$-form $\Omega=\left(\prod_{i} x_{i}-\right.$ $1)^{-1} d x_{1} \wedge \cdots \wedge d x_{n}$ defined on $\left(\mathbb{C} P^{1}\right)^{n} \backslash D$, in coordinates charts $y_{i}=1$.

Proposition 4.3 (Auroux). The tori $\Theta_{s}^{n}$ are special Lagrangians [1, Definition 2.1] with respect to $\Omega$

Proof. See [2, Example 3.3.1] and [1, Proposition 5.2].

Also, we clearly have:

Proposition 4.4. We have that $\left(\left(\mathbb{C} P^{1}\right)^{n}, \Theta_{s}^{n}\right)$ is $T^{n-1}$-pseudohomogeneous, for the action (4.1). 
4.2. The Potential of $\Theta_{s}^{n}$. We come back to our Lagrangian tori $\Theta_{s}^{n}$. We would like to describe the potential $\mathfrak{P O}^{L}$ in coordinates of the form $(2.3)$ with respect to a nice basis for $\pi_{2}\left(\left(\mathbb{C} P^{1}\right)^{n}, \Theta_{s}^{n}\right)$. Fix a point $a_{s} \in \gamma_{s}$. Consider the $S^{1}$ action given by the $i$-th coordinate of the $T^{n-1}$ action described in (4.1). Take the orbit lying in $\Theta_{s}^{n} \cap f^{-1}\left(a_{s}\right)$ and consider its parallel transport over the segment $\left[0, a_{s}\right]$, formed by orbits of the considered $S^{1}$ action that collapse to a point over 0 , giving rise to a Lagrangian disk. Define $\alpha_{i} \in \pi_{2}\left(\Theta_{s}^{n},\left(\mathbb{C} P^{1}\right)^{n}\right)$ to be the class of the above disk. Also, from now one we write $\beta=\beta_{\gamma_{s}}$ and $H_{i}=p_{i}^{*}\left[\mathbb{C} P^{1}\right] \in \pi_{2}\left(\left(\mathbb{C} P^{1}\right)^{n}\right)$ the pullback of the class of the line by the $i$-th projection. Note that $\beta, \alpha_{1}, \ldots, \alpha_{n-1}, H_{1}, \ldots, H_{n}$ are generators of $\pi_{2}\left(\left(\mathbb{C} P^{1}\right)^{n}, \Theta_{s}^{n}\right)$. We assume that our monotone symplectic form is so that $\int_{H_{i}} \omega=1$.

Set $u=z_{\beta}$ and $w_{i}=z_{\alpha_{i}}, i \in(1, \ldots, n-1)$. Note that $z_{H_{i}}\left(\nabla^{\prime}\right)=T^{\int_{H_{i}} \omega} \exp \left(b \cap \partial H_{i}\right)=T$.

Proposition 4.5 ([1, 2]). The potential function encoding the count of Maslov index 2 holomorphic disks with boundary on the Lagrangian tori $\Theta_{s}^{n}$ (for some spin structure) is given by

$$
\mathfrak{P O}^{\Theta_{s}^{n}}=u+\frac{T}{u}\left(1+w_{1}+\cdots+w_{n-1}\right)\left(1+\frac{1}{w_{1}}+\cdots+\frac{1}{w_{n-1}}\right)
$$

Idea of proof. First we consider positivity of intersection of an holomorphic disk with the complex submanifolds $\left\{x_{i}=0\right\},\left\{y_{i}=0\right\},\left\{\prod_{i} x_{i}=\prod_{i} y_{i}\right\}$, for all $i \in(1, \ldots, n)$, to conclude that Maslov index 2 classes admitting holomorphic representatives must be of the form $\beta$, $H_{i}-\beta-\alpha_{i}+\alpha_{j}$, where $i, j=1, \ldots, n$ and $\alpha_{n}=0$. Computations of the holomorphic disks and their algebraic count can be done explictly. We omit here since it follows a straightforward procedure as in [1, Proposition 5.12], see final remark after Proposition 3.3 in [2]. See also [21, Section 5] for similar computations.

We can choose a spin structure so that every disk counts positively, i.e., ev $\operatorname{ev}_{0} \mathscr{M}_{1} \rightarrow \Theta_{s}^{n}$ is orientation preserving, e.g. by choosing a trivialisation of $T \Theta_{s}^{n}$ using the boundary of $\left\{\alpha_{1}, \cdots, \alpha_{n-1}, \beta\right\}$, as spin structure. See [21, Section 5.5] and [?, Section 8], for a complete discussion in a similar scenario.

Remark 4.6. The potential of $\Theta_{s}^{n}$ can be obtained from the known potential for the Clifford torus, $\underset{n}{\times} S_{\text {eq. }}^{1}$. It is given by

$$
\mathfrak{P O}^{\text {Clif }}=z_{1}+\cdots+z_{n}+\frac{T}{z_{1}}+\cdots+\frac{T}{z_{n}} .
$$

We obtain the potential for $\Theta_{s}^{n}$ via wall-crossing transformation $u=z_{n}\left(1+w_{1}+\cdots w_{n-1}\right)$, $w_{i}=z_{i} / z_{n}$. See [2, Example 3.3.1].

Proposition 4.7. The tori $\Theta_{s}^{n}$ satisfy Assumption 2.1, with respect to the standard complex structure of $\left(\mathbb{C} P^{1}\right)^{n}$.

Proof. To prove Assumption $\left(A_{1}\right)$ we use similar argument as in [1, Example 3.3.1]. First we use that $\Theta_{s}^{n}$ are special Lagrangians, and hence, by [1, Lemma 3.1], the Maslov index is twice the intersection with the divisor $D$. This shows that $\mu_{\Theta_{s}^{n}}(\beta) \geq 0, \forall \beta \in \pi_{2}\left(\left(\mathbb{C} P^{1}\right)^{n}, \Theta_{s}^{n}\right)$ represented by an holomorphic disk $u$. Now, if $u$ is a Maslov index 0 holomorphic disk, then $f \circ u$ is well define and lies in $\mathbb{C} \backslash\{1\}$, hence it is a constant in $\gamma_{s}$. Since the regular fibres of $f$ are diffeomorphic to $\left(\mathbb{C}^{*}\right)^{n-1}$, we have that $u$ is itself is constant.

The proof of Assumption $\left(A_{2}\right)$ follows from $\left(\left(\mathbb{C} P^{1}\right)^{n}, \Theta_{s}^{n}\right)$ being $T^{n-1}$-pseudohomogeneous together with Lemma 3.2 . We just need to check that since the $T^{n-1}$-orbit in $\Theta_{s}^{n}$ is generated 
by $\partial \alpha_{i}$, therefore transverse to the boundary of the Maslov index 2 disks with boundary in $\Theta_{s}^{n}$, whose relative homotopy classes are $\beta$ and $H_{i}-\beta-\alpha_{i}+\alpha_{j}, i, j=1, \ldots, n$ and $\alpha_{n}=0$.

4.3. Regarding Proposition 1.4, and Conjecture 1.5. We start noting that Maslov index 2 classes in $H_{2}\left(\left(\mathbb{C} P^{1}\right)^{n}, \Theta_{s}^{n} ; \mathbb{Z}\right)$ are of the form

$$
\beta+k_{1}\left(H_{1}-2 \beta\right)+\cdots+k_{n}\left(H_{n}-2 \beta\right)+l_{1} \alpha_{1}+\cdots+l_{n-1} \alpha_{n-1},
$$

where $\beta$ is the Maslov index 2 and $\alpha_{i}$ the Maslov index 0 classes described in Section 4.2, viewed in $H_{2}\left(\left(\mathbb{C} P^{1}\right)^{n}, \Theta_{s}^{n} ; \mathbb{Z}\right)$ via $\pi_{2}\left(\left(\mathbb{C} P^{1}\right)^{n}, \Theta_{s}^{n}\right) \hookrightarrow H_{2}\left(\left(\mathbb{C} P^{1}\right)^{n}, \Theta_{s}^{n} ; \mathbb{Z}\right)$. Recalling that $\int_{H_{i}} \omega=1$ and $\int_{\alpha_{i}} \omega=0$, we see that area of Maslov index 2 disks belongs to $\{s+(1-2 s) \mathbb{Z}\} \subset \mathbb{R}$.

Proof of Proposition 1.4. We note that each torus

$$
\Theta_{s_{1}}^{k_{1}} \times \cdots \times \Theta_{s_{l}}^{k_{l}} \times\left(S_{\text {eq }}^{1}\right)^{n-\sum_{i} k_{i}}
$$

bounds a disk of Maslov index 2 and symplectic area 1/2, if $n>\sum_{i} k_{i}$, coming from a Maslov index 2 disk in the last $\mathbb{C} P^{1}$ factor, with boundary in its equator $S_{\text {eq }}^{1}$. We see that $1 / 2$ is in $\{s+(1-2 s) \mathbb{Z}\}$ if and only if $s=1 / 2$. This rules out the possibility of $\Theta_{s_{1}}^{k_{1}} \times \cdots \times \Theta_{s_{l}}^{k_{l}} \times\left(S_{\text {eq }}^{1}\right)^{n-\sum_{i} k_{i}}$ being symplectomorphic to $\Theta_{s}^{n}$ for $s \neq 1 / 2$.

For $s=1 / 2$ the torus $\Theta_{s}^{n}$ is monotone, hence the Maslov index $2 J$-holomorphic disks becomes an invariant of its symplectomorphism class - this was first pointed out in [8], see also [21, Theorem 6.4]. This invariant allows us to distinguish between (the symplectomorphism classes of) $\Theta_{s}^{n}$ and $\Theta_{s_{1}}^{k_{1}} \times \cdots \times \Theta_{s_{l}}^{k_{l}} \times\left(S_{\text {eq }}^{1}\right)^{n-\sum_{i} k_{i}}$. For instance, one could look for pairs $\left(\sigma_{1}, \sigma_{2}\right)$ of (relative homotopy classes represented by) Maslov index 2 holomorphic disks with $\partial \sigma_{1}=-\partial \sigma_{2}$. For the torus $\Theta_{s}^{n}$, we must have $\partial \sigma_{i}= \pm \partial \beta$, i.e., only one possibility for $\partial \sigma_{i}$ modulo sign, see Proposition 4.5. But for each torus $\Theta_{s_{1}}^{k_{1}} \times \cdots \times \Theta_{s_{l}}^{k_{l}} \times\left(S_{\text {eq }}^{1}\right)^{n-\sum_{i} k_{i}}$ we have more than one possibility for $\dot{\partial} \sigma_{i}$, modulo sign.

Remark 4.8. Note that, by Proposition 4.5, the total number of Maslov index 2 holomorphic disks with boundary in $\Theta_{s}^{n}$ is $1+n^{2}$, while for the tori $\Theta_{s_{1}}^{k_{1}} \times \cdots \times \Theta_{s_{l}}^{k_{l}} \times\left(S_{\text {eq }}^{1}\right)^{n-\sum_{i} k_{i}}$ it is $\sum_{i=1}^{l}\left(1+k_{i}^{2}\right)+2\left(n-\sum_{i=1}^{l} k_{i}\right)=2 n+\sum_{i=1}^{l}\left(k_{i}-1\right)^{2}$. Hence they can be equal if $(n-1)^{2}=\sum_{i=1}^{l}\left(k_{i}-1\right)^{2}$.

Remark 4.9. The above argument also proves the monotone version $(s=1 / 2)$ of Conjecture 1.5 .

We proceed now to show that holomorphic disks with boundary in $\Theta_{s}^{n}$ with Maslov index bigger than 2 have area bigger than $a=1-s$ - the minimal area of Maslov index 2 holomorphic disks for $s>1 / 2$.

Proposition 4.10. For $k>0$ and $s \in[1 / 2,1)$, the area of holomorphic Maslov index $2 k$ disk with boundary on $\Theta_{s}^{n}$ is least $1-s$, with respect to the standard complex structure in $\left(\mathbb{C} P^{1}\right)^{n}$. The minimum only occur if $k=1$.

Proof. Maslov index $2 k$ disks are in relative classes of the form

$$
k \beta+k_{1}\left(H_{1}-2 \beta\right)+\cdots+k_{n}\left(H_{n}-2 \beta\right)+l_{1} \alpha_{1}+\cdots+l_{n-1} \alpha_{n-1} .
$$

If they are represented by holomorphic disks, their intersection with the divisors $\left\{y_{i}=0\right\}$ and $\left\{\prod_{i=1}^{n} x_{i}=\prod_{i=1}^{n} y_{i}\right\}=\overline{\left\{f^{-1}(1)\right\}}$ is non-negative - recall from Definitions 4.1, 4.2 that 1 is in the interior of $\gamma \subset \mathbb{C}^{*}$. Noting that

$$
\beta \cdot\left\{y_{i}=0\right\}=0, \quad \alpha_{j} \cdot\left\{y_{i}=0\right\}=0, \quad H_{j} \cdot\left\{y_{i}=0\right\}=\delta_{i j},
$$


and

$$
\beta \cdot \overline{\left\{f^{-1}(1)\right\}}=1, \quad \alpha_{j} \cdot \overline{\left\{f^{-1}(1)\right\}}=0, \quad H_{j} \cdot \overline{\left\{f^{-1}(1)\right\}}=1,
$$

$i, j=1, \ldots, n$, we get that

$$
k_{i} \geq 0 \forall i=1, \ldots, n \text { and } k-\sum_{i=1}^{n} k_{i} \geq 0 .
$$

The result follows from taking the symplectic area of (4.4), which is

$$
k s+\sum_{i=1}^{n} k_{i}(1-2 s)=s\left(k-\sum_{i=1}^{n} k_{i}\right)+(1-s)\left(\sum_{i=1}^{n} k_{i}\right)
$$

As pointed out before the above Proposition allows us to informally argue why Conjecture 1.5 should hold. Indeed, for $s>1 / 2$, the number of Maslov index 2 holomorphic disks with boundary in $\Theta_{s}^{n}$ and with minimal area $a=1-s$ is $n^{2}$, by Proposition 4.5. Hence the number of Maslov index 2 disks with boundary in $\Theta_{s_{1}}^{k_{1}} \times \cdots \times \Theta_{s_{l}}^{k_{l}}$ and with minimal area is at most $\sum_{i=1}^{l} k_{i}^{2}<\left(\sum_{i=1}^{l} k_{i}\right)^{2}=n^{2}$, for $l>1$.

\section{Proof of Theorem 1.1 - Bulk deformations}

In this section we use bulk deformations to prove that the tori $\Theta_{s}^{n}$ are non-displaceable for $n$ even and $s \in[1 / 2,1)$, as done in [16] for the case $n=2$. In [16], Fukaya-Oh-Ohta-Ono used the cocycle Poincaré dual to the anti-diagonal in $\mathbb{C} P^{1} \times \mathbb{C} P^{1}$ to bulk-deform Floerhomology. In this section we will bulk-deform Floer-homology by an element of the form $T^{\rho}[h] \in H^{*}\left(\left(\mathbb{C} P^{1}\right)^{n}, \Lambda_{+}\right)$, where $[h] \in H^{2}\left(\left(\mathbb{C} P^{1}\right)^{n}, \mathbb{Z}\right)$.

For $1 \leq i \leq n$, let $h_{i}$ be the cocycle Poincaré dual to $\left\{y_{i}=0\right\} \subset\left(\mathbb{C} P^{1}\right)^{n}$.

Proposition 5.1. The potential for the Lagrangian tori $\Theta_{s}^{n}$, bulk deformed by the cocycle $\mathfrak{b}=T^{\rho}\left[\left(k_{1}+k_{n}\right) h_{1}+\cdots+\left(k_{n-1}+k_{n}\right) h_{n-1}+k_{n} h_{n}\right] \in C^{2}\left(\left(\mathbb{C} P^{1}\right)^{n}, \Lambda_{+}\right)$is given by

$$
\mathfrak{P} \mathfrak{O}_{\mathfrak{b}}^{\Theta_{s}^{n}}(b)=u+\frac{T}{u}\left(1+w_{1}+\cdots+w_{n-1}\right)\left(1+\frac{e^{k_{1} T^{\rho}}}{w_{1}}+\cdots+\frac{e^{k_{n-1} T^{\rho}}}{w_{n-1}}\right) e^{k_{n} T^{\rho}}
$$

Proof. The relative classes $\beta, \alpha_{j}$ have no intersection with $\left\{y_{k}=0\right\}$ viewed as a cycle in $\left(\mathbb{C} P^{1}\right)^{n} \backslash \Theta_{s}^{n}$. Therefore the disk in the class $H_{i}-\beta-\alpha_{i}+\alpha_{j}$ intersect $\left\{y_{k}=0\right\}$ if and only if $k=i$, and with multiplicity 1 . Hence, the coefficient of the monomial $T w_{j} / u w_{i}$ is bulk-deformed by $\mathfrak{b}_{s}$ to $e^{\left(k_{i}+k_{n}\right) T^{\rho}}$.

Lemma 5.2. The potential for the Lagrangian tori $\Theta_{s}^{n}$, bulk deformed by the cocycle $\mathfrak{b}=$ $T^{\rho}\left[\left(k_{1}+k_{n}\right) h_{1}+\cdots+\left(k_{n-1}+k_{n}\right) h_{n-1}+k_{n} h_{n}\right] \in C^{2}\left(\left(\mathbb{C} P^{1}\right)^{n}, \Lambda_{+}\right)$have its critical points given by:

$$
w_{i}=\epsilon_{i} e^{\frac{k_{i}}{2} T^{\rho}}, u=\epsilon_{n} e^{\frac{k_{n}}{2} T^{\rho}} T^{\frac{1}{2}}\left(1+\sum_{i \geq 1}^{n-1} \epsilon_{i} e^{\frac{k_{i}}{2} T^{\rho}}\right),
$$

where $\epsilon_{i}= \pm 1$. 
Proof. For easier notation, let $b_{i}=e^{k_{i} T^{\rho}}$. Taking the differential of the bulk deformed potential $\mathfrak{P O}_{\mathfrak{b}}^{\Theta_{s}^{n}}(b)$ with respect to $w_{i}$ and equating to 0 , we get, after multiplying by $w_{i}$, equations

$$
(i): w_{i}+\sum_{j \neq i} \frac{b_{j} w_{i}}{w_{j}}-b_{i}\left(\frac{1}{w_{i}}+\sum_{j \neq i} \frac{w_{j}}{w_{i}}\right)=0 .
$$

Summing all the equations $(1), \ldots,(n)$, we end up with

$$
\sum_{i=1}^{n-1} w_{i}-\sum_{i=1}^{n-1} \frac{b_{i}}{w_{i}}=0
$$

Let

We have that

$$
L=\sum_{i=1}^{n-1} w_{i}=\sum_{i=1}^{n-1} \frac{b_{i}}{w_{i}}
$$

$$
\begin{aligned}
w_{i} L-b_{i} & =\sum_{j \neq i} \frac{b_{j} w_{i}}{w_{j}}, \\
\frac{L}{w_{i}}-1 & =\sum_{j \neq i} \frac{w_{j}}{w_{i}} .
\end{aligned}
$$

Substituting the above into equations $(i)$ (see (5.1)), we get that

$$
\left(w_{i}-\frac{b_{i}}{w_{i}}\right)(1+L)=0
$$

So if $u, w_{1}, \ldots w_{n-1}$ are critical points of the bulk deformed potential $\mathfrak{P O}_{\mathfrak{b}}^{\Theta_{s}^{n}}(b)$, besides equation (5.2), we must have

$$
\partial_{u} \mathfrak{P O}_{\mathfrak{b}}^{\Theta_{s}^{n}}=1-\frac{b_{n} T}{u^{2}}(1+L)^{2}=0
$$

Hence $L \neq-1$, and therefore

$$
w_{i}=\sqrt{b_{i}}=\epsilon_{i} e^{\frac{k_{i}}{2} T^{\rho}}, u=\sqrt{b_{n}} T^{\frac{1}{2}}(1+L)=\epsilon_{n} e^{\frac{k_{n}}{2} T^{\rho}} T^{\frac{1}{2}}\left(1+\sum_{i \geq 1}^{n-1} \epsilon_{i} e^{\frac{k_{i}}{2} T^{\rho}}\right),
$$

We call the valuation of an element in $\Lambda_{+}$the smallest exponent with non-zero coefficient. Looking at the expression of the critical points of the previous Lemma, one can see that:

Lemma 5.3. Looking at the critical points given on Lemma 5.2 we have that, the valuation of $u$ is not $1 / 2$ if and only if $n=2 m$ and $m-1 \epsilon_{i}$ 's are equal to 1 while the other $m \epsilon_{i}$ 's are equal to -1 , where $i=1, \ldots, 2 m-1$. In that case, the valuation of $u$ is $T^{1 / 2+\rho}$, provided $\sum_{i=1}^{2 m-1} \epsilon_{i} k_{i} \neq 0$.

Now we recall that

$$
u=z_{\beta}=T^{s} \exp (b \cap \partial \beta)
$$

for the class $\beta$ defined in the beginning of Section 4.2. By Lemma 5.3, we have: 
Corollary 5.4. Take $s>1 / 2$ and consider the cocycle $\mathfrak{b}_{s}=T^{s-1 / 2}\left[\left(k_{1}+k_{2 m}\right) h_{1}+\cdots+\right.$ $\left.\left(k_{2 m-1}+k_{2 m}\right) h_{2 m-1}+k_{2 m} h_{2 m}\right] \in C^{2}\left(\left(\mathbb{C} P^{1}\right)^{2 m}, \Lambda_{+}\right)$. Assume that not all $k_{i}$ 's are 0 , for $i=1, \ldots, 2 m-1$, i.e., $\left[\mathfrak{b}_{s}\right]$ is not a multiple of the monotone symplectic form. Then there exists $b_{s}$ a critical point of $\mathfrak{P O}_{\mathfrak{b}_{s}}^{\Theta_{s}^{2 m}}$.

Recalling that $\Theta_{s}^{2 m}$ satisfy Assumption 2.1 (Propositions 4.7), for some almost complex structure $J$, and noting that $\Theta_{s}^{2 m}$ is a contractible Lagrangian torus of $\left(\mathbb{C} P^{1}\right)^{2 m}$, we have that $\left(\left(\mathbb{C} P^{1}\right)^{2 m}, \Theta_{s}^{2 m}\right)$ satisfy all the hypothesis of Corollary 2.8. Therefore, from Corollaries 2.8 and 5.4, we deduce:

Theorem 5.5. For $s \geq 1 / 2$ there exists a bulk $\left[\mathfrak{b}_{s}\right] \in H^{2}\left(\left(\mathbb{C} P^{1}\right)^{2 m}, \Lambda_{+}\right)$and a weak bounding cochain $b_{s} \in H^{1}\left(\Theta_{s}^{2 m}, \Lambda_{0}\right)$ such that $H F\left(\Theta_{s}^{2 m},\left(b_{s}, \mathfrak{b}_{s}\right) ; \Lambda_{0, \text { nov }}\right) \cong H\left(\Theta_{s}^{2 m}, \Lambda_{0, \text { nov }}\right)$.

This proves the first part of Theorem 1.6. Theorem 1.1 follows from Theorem 2.4 and Theorem 5.5.

Corollary 1.3 follows from the same arguments as above using that

$$
\mathfrak{P O}_{\mathfrak{b}}^{\Theta_{s_{1}}^{k_{1}} \times \cdots \times \Theta_{s_{l}}^{k_{l}} \times\left(S_{\mathrm{eq}}^{2}\right)^{n-\sum_{i} k_{i}}}=\mathfrak{P} \mathfrak{O}_{\mathfrak{b}}^{\Theta_{s_{1}}^{k_{1}}}+\cdots+\mathfrak{P O}_{\mathfrak{b}}^{\Theta_{s_{l}}^{k_{l}}}+\mathfrak{P O}_{\mathfrak{b}}^{\left(S_{\mathrm{eq}}^{2}\right)^{n-\sum_{i} k_{i}}}
$$

\section{QUASI-MORPHISMS AND QUASI-STATES}

In this section we prove the last part of Theorem 1.6. It follows arguments similar to [15, Theorem 23.4].

Lemma 6.1. For any $\mathfrak{b}=T^{\rho}\left[l_{1} h_{1}+\cdots+l_{n-1} h_{n-1}+l_{n} h_{n}\right] \in C^{2}\left(\left(\mathbb{C} P^{1}\right)^{n}, \Lambda_{+}\right)$, the bulk deformed Quantum cohomology [15, Section 5] is semi-simple.

Proof. By [17, Theorem 1.1.1] (see also [12, Theorem 6.1], for the Fano case) we have an isomorphism between the bulk deformed Quantum cohomology of a toric symplectic manifold and the Jacobian Ring of the bulk deformed toric potential. If the bulk deformed toric potential has only non-degenerate critical points, we can split the Quantum cohomology ring into orthogonal algebra summands according to the factors corresponding to the critical points under the isomorphism given in [17, Theorem 1.1.1].

Naming now $z_{i}=z_{\beta_{i}}(2.3)$, for $\beta_{i}$ the class of Maslov index 2 holomorphic disk intersecting $\left\{x_{i}=0\right\}$, we have that the bulk deformed potential of a toric fiber is:

$$
\mathfrak{P O}_{\mathfrak{b}}=z_{1}+\cdots+z_{n}+\frac{T e^{l_{1} T^{\rho}}}{z_{1}}+\cdots+\frac{T e^{l_{n} T^{\rho}}}{z_{n}},
$$

whose critical points are given by $\left(z_{1}, \ldots, z_{n}\right)=\left(\epsilon_{1} T^{1 / 2} e^{l_{1} T^{\rho} / 2}, \ldots, \epsilon_{n} T^{1 / 2} e^{l_{n} T^{\rho} / 2}\right)$. Hence, there are $2^{n}$ idempotents of $Q H_{\mathfrak{b}}\left(\left(\mathbb{C} P^{1}\right)^{n} ; \Lambda_{0, \text { nov }}\right), \mathbf{e}_{1}^{\mathfrak{b}}, \ldots, \mathbf{e}_{2^{n}}^{\mathfrak{b}}$ for which

$$
Q H_{\mathfrak{b}}\left(\left(\mathbb{C} P^{1}\right)^{n} ; \Lambda_{0, \text { nov }}\right)=\bigoplus_{i=1}^{2^{n}} \Lambda_{0, \text { nov }} \mathbf{e}_{i}^{\mathfrak{b}} .
$$

In [15, Section 17, (17.18)], given $X$ a symplectic manifold and $L$ a relatively spin Lagrangian submanifold, Fukaya-Oh-Ohta-Ono construct an homomorphism:

$$
i_{\mathrm{qm},(b, \mathfrak{b})}^{*}: Q H_{\mathfrak{b}}\left(X ; \Lambda_{0, \text { nov }}\right) \rightarrow H F\left(L,(b, \mathfrak{b}) ; \Lambda_{0, \text { nov }}\right),
$$

which is proven to be a ring homomorphism in [?], see [15, Remark 17.16] and [?, Section 4.7]. 
Applying Lemma 6.1 for $\left(\mathbb{C} P^{1}\right)^{2 m}$ and $\mathfrak{b}_{s}$ given in Theorem 5.5, using that $i_{\mathrm{qm},\left(b_{s}, \mathfrak{b}_{s}\right)}^{*}$ is unital and $H F\left(\Theta_{s}^{2 m},\left(b_{s}, \mathfrak{b}_{s}\right) ; \Lambda_{0, \text { nov }}\right) \neq 0$, we have:

Proposition 6.2. There exists an idempotent $\boldsymbol{e}_{s} \in Q H_{\mathfrak{b}_{s}}\left(\left(\mathbb{C} P^{1}\right)^{2 m} ; \Lambda_{0, \text { nov }}\right)$ for which $i_{\mathrm{qm},\left(b_{s}, \mathfrak{b}_{s}\right)}^{*}\left(\boldsymbol{e}_{s}\right) \neq$ 0 in $\operatorname{HF}\left(\Theta_{s}^{2 m},\left(b_{s}, \mathfrak{b}_{s}\right) ; \Lambda_{0, \text { nov }}\right)$.

Theorem 1.6 follows then from Proposition 6.2 and Theorem 18.8 of [15].

\section{TORI IN $\mathbb{C} P^{2} \# 3 \overline{\mathbb{C} P^{2}}$}

In this section we prove Theorem 1.12 . We will describe a model for $\left(\mathbb{C} P^{2} \# 3 \overline{\mathbb{C} P^{2}}, \omega_{\epsilon}\right)=$ $\left(\mathbb{C} P^{1} \times \mathbb{C} P^{1} \# 2 \overline{\mathbb{C} P^{2}}, \omega_{\epsilon}\right)$ which is equivalent to performing two blowups of capacities $\epsilon$ centred at the rank 0 elliptic singularities (corners) of the singular fibration of $\mathbb{C} P^{1} \times \mathbb{C} P^{1}$ described in [16], see Figure 1.
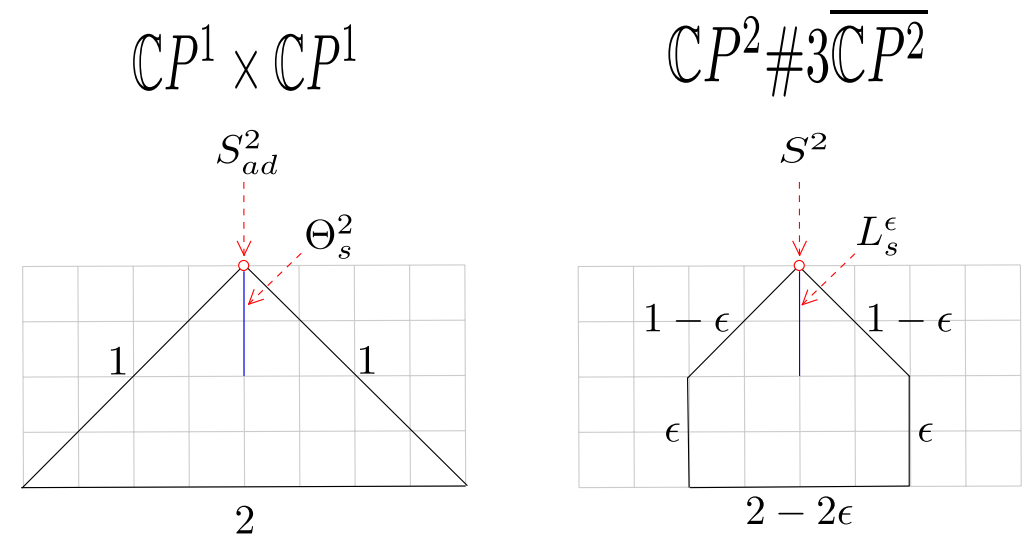

Figure 1. Singular fibrations of $\mathbb{C} P^{1} \times \mathbb{C} P^{1}$ and $\mathbb{C} P^{2} \# 3 \overline{\mathbb{C} P^{2}}$.

Consider $\mathbb{C} P^{1} \times \mathbb{C} P^{1}$ with coordinates $\left(\left[x_{1}: y_{1}\right],\left[x_{2}: y_{2}\right]\right)$ as in Section 4.1. Consider also the tori $\Theta_{s}^{2}$, the function $f=x_{1} x_{2} / y_{1} y_{2}$, the relative class $\beta$ and $\alpha:=\alpha_{1}$ and the divisor $D=f^{-1}(1) \cup\left\{y_{1}=0\right\} \cup\left\{y_{2}=0\right\}$, as defined in Section 4.2 .

From Proposition 4.3 and [1, Lemma 3.1], we have that $2[D] \in H_{2}\left(\mathbb{C} P^{1} \times \mathbb{C} P^{1} \backslash \Theta_{s}^{2}\right)$ is Poincaré dual to the Maslov class $\mu_{\Theta_{s}^{2}} \in H^{2}\left(\mathbb{C} P^{1} \times \mathbb{C} P^{1}, \Theta_{s}^{2}\right)$. In particular the Maslov index 2 holomorphic disks, computed in Proposition 4.5 for $n=2$, do not intersect $\overline{f^{-1}(1)} \cap\left\{y_{1}=\right.$ $0\}=([1: 0],[0: 1])=p_{1}$ and $\overline{f^{-1}(1)} \cap\left\{y_{2}=0\right\}=([0: 1],[1: 0])=p_{2}$.

Let $B_{i}(\epsilon)$ be the ball of capacity [18, Section 12] $\epsilon$ (radius $\sqrt{\epsilon / \pi}$ ) centered at $p_{i}$, in the coordinate plane $x_{i}=1, y_{j}=1, i, j=1,2, i \neq j$. Denote $S_{i}(\epsilon)=\partial B_{i}(\epsilon)$. Let $\left(\mathbb{C} P^{2} \# 3 \overline{\mathbb{C} P^{2}}, \omega_{\epsilon}\right)$ be the result of blowing up [18, Section 7] $\mathbb{C} P^{1} \times \mathbb{C} P^{1}$ with respect to $B_{1}(\epsilon)$ and $B_{2}(\epsilon)$, so that the exceptional curves $E_{i}$ (coming from collapsing the Hopf fibration in $S_{i}(\epsilon)$ ) have symplectic area $\omega_{\epsilon}\left(E_{i}\right)=\epsilon, i=1,2$. Let $j_{\epsilon}$ be the induced complex structure and $L_{s}^{\epsilon}$ correspond to $\Theta_{s}^{2}$ after the blowup. Note that $\epsilon$ can take any value in $(0,1)$, so that $B_{1}(\epsilon) \cap B_{2}(\epsilon)=\emptyset$.

Note also that $f=x_{1} x_{2} / y_{1} y_{2}$ is constant along the fibers of the Hopf fibration of both $S_{1}(\epsilon)$ and $S_{2}(\epsilon)$. In particular it give rise to a $\left(j_{\epsilon}, j\right)$-holomorphic function $\tilde{f}: \mathbb{C} P^{2} \# 3 \overline{\mathbb{C} P^{2}} \rightarrow \mathbb{C} P^{1}$.

For computing the potential for $L_{s}^{\epsilon}$ it is interesting that the disks of Proposition 4.5, remain essentially the same. This can be obtained by stretching the complex structure $j_{\epsilon}$. So take $\delta$ small enough so that $B_{1}(\delta) \cup B_{2}(\delta)$ does not intersect any Maslov index 2 holomorphic disk. 
Consider a diffeomorphism $\varphi:\left(\mathbb{C} P^{2} \# 3 \overline{\mathbb{C} P^{2}}, \omega_{\epsilon}\right) \rightarrow\left(\mathbb{C} P^{2} \# 3 \overline{\mathbb{C} P^{2}}, \omega_{\delta}\right)$ coming from a finite neck stretch [7, 4] along $S_{i}\left(\epsilon+\delta^{\prime}\right) \subset\left(\mathbb{C} P^{2} \# 3 \overline{\mathbb{C} P^{2}}, \omega_{\epsilon}\right)$ [4, 17, see also [22, Section 3], which sends $L_{s}^{\epsilon}$ to $L_{s}^{\delta}$. The diffeomorphism $\varphi$ is equivalent to considering an inflation along the exceptional curves $E_{i}, i=1,2$. Set $J_{\delta}=\varphi^{*} j_{\delta}$, an $\omega_{\epsilon}$ compatible almost complex structure.

Lemma 7.1. We have that $\left(\mathbb{C} P^{2} \# 3 \overline{\mathbb{C} P^{2}}, L_{s}^{\epsilon}, J_{\delta}\right)$ satisfy Assumption 2.1. The potential function for $L_{s}^{\epsilon}$ with respect to $J_{\delta}$, is given by:

$$
\mathfrak{P O}^{L_{s}^{\epsilon}}=u+\frac{T}{u}(1+w)\left(1+\frac{1}{w}\right)+T^{1-\epsilon}\left(w+\frac{1}{w}\right)
$$

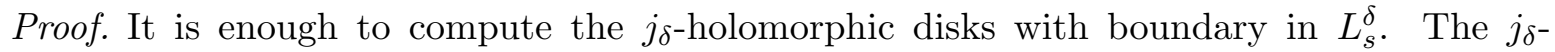
holomorphic disks that don't intersect the exceptional divisors $E_{1}, E_{2}$, corresponds to the holomorphic disks in $\mathbb{C} P^{1} \times \mathbb{C} P^{1}$ with boundary in $\Theta_{s}^{2}$, which gives the terms

$$
u+\frac{T}{u}(1+w)\left(1+\frac{1}{w}\right)
$$

of $\mathfrak{P O}^{L_{s}^{\delta}}$, and are regular.

Let $\tilde{D}$ be the proper transform of the divisor $D \in \mathbb{C} P^{1} \times \mathbb{C} P^{1}$. It can be checked that, twice $\tilde{D}+E_{1}+E_{2}$ is Poincaré dual to the Maslov class $\mu_{L_{s}^{\delta}}$. This implies Assumption $\left(A_{1}\right)$ as in the proof of Proposition 4.7. Moreover, Maslov index 2 disks intersects $\tilde{D}+E_{1}+E_{2}$ once. Which means that if a $j_{\delta}$-holomorphic disk $u$ intersects either $E_{1}$ or $E_{2}$, by positivity of intersection, it does not intersect $\tilde{D}$ and hence $\tilde{f} \circ u: \mathbb{D} \rightarrow \mathbb{C}^{*}$ must be constant. There are two Maslov index 2 disks in the fiber $\tilde{f}^{-1}(c)$, for $c \in \gamma_{s}$. Looking at the intersections with $E_{i}$, and the proper transform of $\left\{x_{i}=0\right\}$ and $\left\{y_{i}=0\right\}$, we can see that the relative classes of these disks are $H_{1}-E_{1}+\alpha$ and $H_{2}-E_{2}-\alpha$ (for some orientation of $\alpha$ ). Since, $\omega_{\epsilon}\left(H_{i}-E_{i} \pm \alpha\right)=1-\epsilon$, we get the remaining term

$$
T^{1-\epsilon}\left(w+\frac{1}{w}\right)
$$

To show regularity of the above disks, one notes that the pre-image under $\tilde{f}$ of a small neighbourhood $\mathcal{N}_{s}$ of $\gamma_{s}$ contain the whole family of the above disks and is actually toric. Moreover, $\left(\tilde{f}^{-1}\left(\mathcal{N}_{s}\right), L_{s}^{\delta}\right)$ is $T^{2}$-homogeneous [11, or if you will, $S^{1}$-pseudohomogeneous (Definition 3.1 ) for a $j_{\delta}$-holomorphic $S^{1}$-action transverse to $\partial \alpha$, which shows Assumption $\left(A_{2}\right)$.

The choice of spin structure is given by trivialising $T L_{s}^{\epsilon}$ according to $\{\alpha, \beta\}$ and is so that the evaluation map is orientation preserving, as in the proof of Proposition 4.5. See also [21, Section 5.5] and [?, Section 8].

Remark 7.2. The above potential can also be computed by a technique similar to the one developed in [16] and also by some gluing procedure similar to the one developed in Section 5.2 of the ArXiv.1002.1660v1 version of [16] and in [24].

Remark 7.3. For each $\delta^{\prime}>0$, the family $\left\{L_{s}^{\epsilon}: s \in\left[1 / 2,1-\delta^{\prime}\right]\right\}$ can be seen as fibres of an almost toric fibration (ATF) of $\mathbb{C} P^{2} \# 3 \overline{\mathbb{C} P^{2}}$, represented by an almost toric base diagram (ATBD) analogous to the one in Figure $9\left(A_{3}\right)$ of [23]. In fact, the singular fibration described by the second diagram in Figure 1 can be thought as a limit of ATFs described by sliding nodes of the ATBD in Figure $9\left(A_{3}\right)$ of $[23]$. Moreover, the potential $\mathfrak{P O}^{L_{s}^{\epsilon}}$ can be obtained from the toric potential

$$
\mathfrak{P O}^{\text {toric }}=u_{1}+u_{2}+\frac{T}{u_{1}}+\frac{T}{u_{2}}+\frac{T^{1-\epsilon} u_{1}}{u_{2}}+\frac{T^{1-\epsilon} u_{2}}{u_{1}},
$$


via wall-crossing transformation $u=u_{1}(1+w), w=u_{2} / u_{1}$, giving another example where actual computations meet wall-crossing predictions [1, 2, 21].

Let $\mathfrak{s} \in C^{2}\left(\mathbb{C} P^{2} \# 3 \overline{\mathbb{C} P^{2}}\right)$ be the cocycle Poincaré dual to $\left\{y_{1}=0\right\} \cup E_{1}$, so $[\mathfrak{s}]=H_{1}-E_{2}+E_{1}$. Analogous to Proposition 5.1, we have:

Proposition 7.4. The potential for $L_{s}^{\epsilon}$, bulk deformed by the cocycle $\mathfrak{b}=T^{\rho} \mathfrak{s} \in C^{2}\left(\mathbb{C} P^{2} \# 3 \overline{\mathbb{C} P^{2}}, \Lambda_{+}\right)$ is given by:

$$
\mathfrak{P O}_{\mathfrak{b}}^{L_{s}^{\epsilon}}=u+\frac{T}{u}(1+w)\left(e^{T^{\rho}}+\frac{1}{w}\right)+T^{1-\epsilon}\left(e^{T^{\rho}} w+\frac{1}{w}\right) .
$$

We can then compute the critical points of $\mathfrak{P O}_{\mathfrak{b}}^{L_{s}^{\epsilon}}$ and obtain:

Lemma 7.5. We have that $w=-e^{\frac{-T^{\rho}}{2}}$ and $u= \pm T^{\frac{1}{2}}\left(1-e^{\frac{-T^{\rho}}{2}}\right)^{\frac{1}{2}}\left(e^{T^{\rho}-e^{\frac{T^{\rho}}{2}}}\right)^{\frac{1}{2}}$ are critical points of $\mathfrak{P O}_{\mathfrak{b}}^{L_{s}^{\epsilon}}$. The valuations of $w$ and $u$ are respectively 0 and $1 / 2+\rho$.

Since we have that $\int_{\beta} \omega_{\epsilon}=s$ and $\int_{\alpha} \omega_{\epsilon}=0$ :

Lemma 7.6. For $s>1 / 2$ and $\mathfrak{b}_{s}^{\epsilon}=T^{s-1 / 2}[\mathfrak{s}]$, there exists a weak bounding cochain $b_{s}^{\epsilon} \in$ $H^{1}\left(L_{s}^{\epsilon}, \Lambda_{0}\right)$ which is a critical point of $\mathfrak{P O}_{\mathfrak{b}_{s}^{\epsilon}}^{L_{s}^{\epsilon}}$.

Following similar arguments as in Sections 5 and 6, we are able to prove Theorem 1.12 and consequently Theorem 1.10 .

\section{REFERENCES}

[1] D. Auroux. Mirror symmetry and T-duality in the complement of an anticanonical divisor. J. Gökova Geom. Topol., 1:51-91, 2007.

[2] D. Auroux. Special Lagrangian fibrations, wall-crossing, and mirror symmetry. In Surveys in differential geometry. Vol. XIII. Geometry, analysis, and algebraic geometry: forty years of the Journal of Differential Geometry, volume 13 of Surv. Differ. Geom., pages 1-47. Int. Press, Somerville, MA, 2009.

[3] P. Biran and O. Cornea. A Lagrangian Quantum Homology. In New Perspectives and Challenges in Symplectic Field Theory, volume 49 of CRM Proc. Lecture Notes, pages 1-44. CRM-AMS, 2009.

[4] F. Bourgeois, Y. Eliashberg, H. Hofer, K. Wysocki, and E. Zehnder. Compactness results in Symplectic Field Theory. Geom. Topol., 7:799-888, 2003.

[5] L. Buhovsky. The Maslov class of Lagrangian tori and quantum products in Floer cohomology. J. Topol. Anal., 2(1):57-75, 2010.

[6] Y. Chekanov and F. Schlenk. Notes on monotone Lagrangian twist tori. Electron. Res. Announc. Math. Sci., 17:104-121, 2010.

[7] Y. Eliashberg, A. Givental, and H. Hofer. Introduction to Symplectic Field Theory. In Visions in Mathematics, pages 560-673. Birkhäuser, 2010.

[8] Y. Eliashberg and L. Polterovich. Unknottedness of Lagrangian surfaces in symplectic 4-manifolds. Internat. Math. Res. Notices, (11):295-301, 1993.

[9] M. Entov and L. Polterovich. Calabi quasimorphism and quantum homology. Int. Math. Res. Notices, 30:1635-1676, 2003.

[10] M. Entov and L. Polterovich. Quasi-states and symplectic intersections. Comment. Math. Helv., 81(1):75-99, 2006.

[11] J. D. Evans and Y. Lekili. Floer cohomology of the Chiang Lagrangian. Selecta Math., 21(4):1361-1404, 2015.

[12] K. Fukaya, Y.-G. Oh, H. Ohta, and K. Ono. Lagrangian Floer theory on compact toric manifolds, I. Duke Math. J., 151(1):23-175, 2010.

[13] K. Fukaya, Y.-G. Oh, H. Ohta, and K. Ono. Lagrangian Intersection Floer Theory: Anomaly and Obstruction, volume 46 of Stud. Adv. Math. American Mathematical Society, International Press, 2010.

[14] K. Fukaya, Y.-G. Oh, H. Ohta, and K. Ono. Lagrangian Floer theory on compact toric manifolds II: bulk deformations. Selecta Math. (N.S.), 17(2):609-711, 2011. 
[15] K. Fukaya, Y.-G. Oh, H. Ohta, and K. Ono. Spectral invariants with bulk quasimorphisms and Lagrangian Floer theory. arXiv:1105.5123, 2011.

[16] K. Fukaya, Y.-G. Oh, H. Ohta, and K. Ono. Toric Degeneration and Nondisplaceable Lagrangian Tori in $S^{2} \times S^{2}$. Internat. Math. Res. Notices, 13:29422993, 2012.

[17] K. Fukaya, Y.-G. Oh, H. Ohta, and K. Ono. Lagrangian Floer theory and mirror symmetry on compact toric manifolds. Astérisque, (376):vi+340, 2016.

[18] D. McDuff and D. A. Salamon. Introduction to symplectic topology. Oxford Mathematical Monographs. 1998.

[19] J. Oakley and M. Usher. On certain Lagrangian submanifolds of $S^{2} \times S^{2}$ and $\mathbb{C P}^{n}$. Algebr. Geom. Topol., 16(1):149-209, 2016.

[20] D. Tonkonog and R. Vianna. Low-area Floer theory and non-displaceability. arXiv:1511.00891, 2015.

[21] R. Vianna. On exotic Lagrangian tori in $\mathbb{C P}^{2}$. Geom. Topol., 18(4):2419-2476, 2014.

[22] R. Vianna. Infinitely many exotic monotone Lagrangian tori in $\mathbb{C P}^{2}$. J. Topol., 9(2):535-551, 2016.

[23] R. Vianna. Infinitely many monotone lagrangian tori in del pezzo surfaces. Selecta Mathematica, pages 1-42, 2017.

[24] W. Wu. On an exotic Lagrangian torus in $\mathbb{C} P^{2}$. Compositio Math., 151(7):1372-1394, 2015. 